\title{
Das deutsche Endoprothesenregister - eine unendliche Geschichte?
}

\author{
The German Endoprosthesis Register - A Never-Ending Story?
}

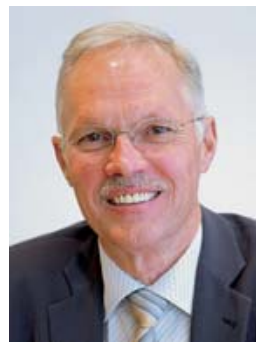

F. U. Niethard

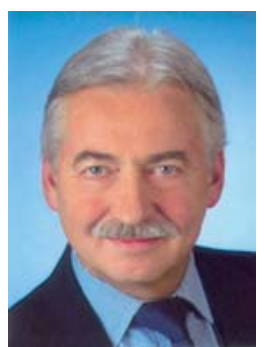

K. Weise

Bibliografie

DOI $10.1055 / \mathrm{s}-0029-1185558$ ZOrthop Unfall 2009; 147: 149150 ๑ Georg Thieme Verlag KG Stuttgart . New York . ISSN 1864-6697

\section{Korrespondenzadressen}

Prof. Dr. med. Fritz U. Niethard Orthopädische Klinik

Universitätsklinik der RWTH Aachen

Pauwelsstraße 30

52074 Aachen

Tel.: 0241/808-9410

Fax: 0241/808-2453

funiethard@

orthopaedie-aachen.de

Prof. Dr. med. Kuno Weise BG-Unfallklinik

Schnarrenbergstraße 95 72076 Tübingen

Tel.: 0 7071/606-1001

Fax: 07071/606-1002

weise@bgu-tuebingen.de
Das schwedische Endoprothesenregister ist der Maßstab aller Dinge, wenn es um Daten zur langfristigen Haltbarkeit von Endoprothesen, aber auch um die frühzeitige Erkennung von Fehlentwicklungen geht. Es existiert seit 30 Jahren und ist somit zum Inbegriff der Qualitätssicherung auf dem Gebiet der Endoprothetik geworden (www.jru.orthop.gu.se). In Deutschland hat es verschiedene Ansätze zur Gründung eines Registers gegeben $[2,9,10]$. Zuletzt war 2007 ein deutsches Register gefordert worden, nachdem ein „Prothesenskandal“ öffentliche Aufmerksamkeit gefunden hatte. Nun ist es wieder still geworden. Labek und Böhler schlagen daher in dieser Zeitschrift die Einrichtung eines europäischen Registers vor [5].

Hat man in Deutschland etwa den Anschluss verpasst? Immerhin war in Deutschland bereits 1994 unter der Leitung von Willert das Deutsche Endoprothesenregister (DER) gegründet worden, das dann schon 1997 unter der Leitung von Kienapfel etwa 7000-8000 Primärimplantationen und 1100 Revisionen am Hüftgelenk sowie 3000 Primärimplantationen und 250 Revisionen am Kniegelenk pro Jahr aus über 40 Kliniken in Deutschland dokumentieren konnte. Das sind kaum weniger als zu diesem Zeitpunkt in Schweden registriert wurden (ca. 13000). Von Anfang an wurde jedoch bemängelt, dass das Langzeitergebnis nicht ermittelt werden konnte, weil hierfür die Beschaffung der notwendigen Daten nicht möglich war.

Im gleichen Zeitraum wurde die Bundesgeschäftsstelle Qualitätssicherung $\mathrm{GmbH}(\mathrm{BQS})$ tätig, um die Versorgungsqualität bei den seinerzeit noch gültigen Fallpauschalen und Sonderentgelten für die Endoprothetik zu erfassen. Mit ursprünglich mehr als 100 „Items“ zur ärztlichen und pflegerischen Versorgung wird bis heute Vieles erfasst, nur eben die Standzeit der Endoprothese nicht. Allerdings konnten aus dem Datenschatz der BQS unterschiedliche Frühergebnisse hinsichtlich der Häufigkeit von Infekten, der Hüftgelenksluxationen und Kniegelenksbeweglichkeit herausgefiltert werden. Deren Relevanz für das Gesamtversorgungssystem wurde immer wieder diskutiert. So konnte es auch nicht verwundern, dass die Einführung einer Mindestmengenregelung für die Knieendoprothetik nicht allein durch Qualitätsaspekte, sondern auch gesundheitspolitisch motiviert war: selbstverständlich wurde von den Kostenträgern auch ein wirtschaftlicher Konzentra- tionsprozess angestrebt, um mit der DRG-Einführung die Fallkosten zu harmonisieren und zu senken [8]. So wurde dann auch vom gemeinsamen Bundesausschuss (G-BA) festgestellt, dass Krankenhäuser, die bei der Kniegelenkstotalendoprothesenoperation die vom G-BA eingeführte Mindestmenge von 50 Eingriffen pro Jahr erfüllen, eine deutliche bessere Behandlungsqualität erzielen als Krankenhäuser, die diese Operationen weniger häufig vornehmen (www.g-ba.de 2008).

Aber auch hierbei handelt es sich um die Beurteilung von Versorgungsindikatoren, und nicht um die Erfassung der Ergebnisqualität. Es bedurfte erst eines „Prothesenskandals“, um die von Vertretern der Fachgesellschaft zahlreich vorgetragenen Initiativen zur Gründung eines Endoprothesenregisters hörbar zu machen. Nachdem zwischen 2006 und 2007 in einem Berliner Krankenhaus Knieendoprothesen fehlerhaft implantiert worden waren, wurde nun auch von der Öffentlichkeit die Forderung des seinerzeitigen Präsidenten der DGOOC, Hassenpflug, gierig aufgegriffen, ein Endoprothesenregister einzurichten.

Doch wo stehen wir heute? Zwei Jahre nach dem „Skandal“ hat sich die Öffentlichkeit wieder beruhigt, denn Endoprothesenoperationen gehören zu den erfolgreichsten Operationen überhaupt. Das belegen die zahlreichen Publikationen in dieser Zeitschrift während der letzten Jahre. Auch in dieser Ausgabe der Zeitschrift für Orthopädie und Unfallchirurgie ist der Endoprothetik breiter Raum gewidmet. Die Artikel beschäftigen sich mit mittelfristigen [1,3] und langfristigen Ergebnissen $[4,7]$ sowie mit der Lösung der Lockerungsproblematik [6]. Doch auch die intensive wissenschaftliche Auseinandersetzung mit der Endoprothetik ersetzt nicht das Endoprothesenregister. Labek und Böhler beschreiben, dass sich in Skandinavien durch die Einführung der Register eindrucksvoll eine Qualitätsverbesserung nachweisen lässt. Darüber hinaus konnte gezeigt werden, dass durch diese Form der Qualitätsüberprüfung eine Kostenreduktion im Gesundheitswesen möglich ist.

Auch die Fachgesellschaften erkennen, dass durch enge Kooperation zwischen Register, Ärzten und Gesundheitsbehörden wesentliche Verbesserungen im Ergebnis endoprothetischer Eingriffe zu erzielen sind. Für die Dokumentation von Langzeitergebnissen müssen die anfallenden Wechseloperationen dokumentiert und bis auf die Erst- 
operation nachverfolgt werden können. Nur so gelingt es, anhand des eingebrachten Implantats Auffälligkeiten bei Material und Implantation zu entdecken. Die anscheinend triviale Forderung nach Datenabgleich bestand unverändert bereits 1997. Die Industrie hat sich bereit erklärt, entsprechende Barcodes einzuführen, die eine Identifikation des Implantats ermöglichen. Nun sind der Datenschutz und vor allem diejenigen Institutionen gefragt, die von gesetzlicher Seite die Qualitätssicherung überwachen. Die Fachgesellschaften haben ihre besondere Bedeutung bei der Interpretation der Ergebnisse hervorgehoben. Sie verweisen damit auf Beispiele aus den skandinavischen Ländern, wo es nur dank der ärztlichen Expertise gelungen ist, Qualitätsverbesserungen zu erzielen. In Ländern, in denen eine ärztliche Mitwirkung nicht besteht, ist dieses Ziel dagegen nicht erreicht worden. In Finnland z.B. ist das Register ohne wesentliche Einbindung der wissenschaftlichen Fachgesellschaften umgesetzt worden. Das finnische Register zeigt aber erheblich schlechtere Rückgänge der Revisionswahrscheinlichkeit als das schwedische, wo die Fachgesellschaften federführend eingebunden sind [5]. Daher wird in Finnland nun die Struktur geändert und die wissenschaftliche Validierung entscheidend gestärkt.

Die Fachgesellschaften sehen mit Sorge, dass derzeit die Einrichtung eines Endoprothesenregisters zwischen BQS oder AQUA oder ggf. auch anderen Instituten hin- und hergereicht wird, während die eigentlich drängenden und fachspezifischen Aspekte im Sinne des Patienten vernachlässigt werden. Da es keinen politischen Konsens bezüglich der Kosten gibt, haben die Asklepios Orthopädische Klinik Lindenlohe und eine Endoprothesenfirma „eine deutschlandweite Vorreiterrolle“ (http://www.endoprothesen-register.com/index.php4?ID_CMS=97) übernommen, um auch der Politik die Notwendigkeit einer Entscheidung zu signalisieren. Natürlich aber auch - wie es heißt - „um die Versorgungsqualität für die Patientinnen und Patienten in dieser Klinik zu verbessern“. Wird nun das deutsche Endoprothesenregister zum Werbeträger privater Klinikketten degradiert und bleibt damit ein für allemal drittrangig?
Nur die wissenschaftlichen Fachgesellschaften haben Zugang zu einer hochwertigen Expertise in der Bewertung der Daten und bieten Strukturen an, um ein Endoprothesenregister auch tatsächlich umzusetzen. Die Endoprothesenindustrie hat sogar ihre Bereitschaft signalisiert, sich an den Kosten zu beteiligen. Qualität und Qualitätskontrolle kosten Geld. Das müssten auch diejenigen Politiker wissen, die sofort nach dem Prothesenskandal nach dem Register gerufen haben. Nun gilt es, Qualität auch zu verwirklichen.

\section{F. U. Niethard}

K. Weise

\section{Literatur}

1 Daniilidis K, Skwara A, Skuginna A et al. Mittelfristiger, klinischer und radiologischer Vergleich von zementierten und zementfreien, medialen, unikompartimentellen Knieendoprothesen Z Orthop Unfall 2009; 147: 188-193

2 Griss P. Qualitätssicherung in der Endoprothetik. Editorial. Z Orthop 1998; 136: 95-96

3 Hauk C, Heyse TJ, Witteborn MCP et al. Matched-Pair-Analyse: Innex FIXUC vs. NexGen LPS. Z Orthop Unfall 2009; 147: 183-187

4 Ishaque BA, Donle E, Gils J et al. 8-Jahres-Resultate der Schenkelhalsprothese ESKA-CUT. Z Orthop Unfall 2009; 147: 158-165

5 Labek G, Böhler N. Das European Arthroplasty Register (EAR), die Entwicklung und Sinnhaftigkeit übernationaler Zusammenarbeit von Endoprothesenregistern. Z Orthop Unfall 2009; 147: 151-157

6 Marx R, Faramarzi R, Jungwirth $F$ et al. Silikatbeschichtung zementierter Titanschäfte für die Reduzierung aseptischer Lockerungsraten. Z Orthop Unfall 2009; 147: 175-182

7 Metzner G, Strassl R, Neumann DRP et al. Langzeitergebnisse der zementfreien Hofer-Imhof-Titan-Schraubpfanne. Z Orthop Unfall 2009; 147: $166-174$

8 Niethard FU, Weise K. Mindestmengen in der Hüftendoprothetik - Qualität durch Quantität? Z Orthop Unfall 2007; 145: 261-262

9 Pitto RP, Lang I, Kienapfel $H$ et al. The German Arthroplasty Register. Acta Orthop Scand Suppl 2002; 73: 30-33

10 Springorum HW. IDEE - das Bad Mergentheimer Endoprothesenregister. Z Orthop 1997; 135: Oa24 